Article original

\title{
Biodiversité végétale et vitesse de la régénération de la forêt classée de Sanaimbo (Côte d'Ivoire)
}

\author{
N’Dja J. KAssi", Emma Ake-Assı \& Marie Solange TIEBRE \\ Laboratoire de Botanique, UFR Biosciences, Université de Cocody-Abidjan 22 BP 582 Abidjan 22 (Côte d'Ivoire) \\ *Auteur pour les correspondances (E-mail : kassindja@yahoo.fr) \\ Reçu le 18-02-2010, accepté le 24-06-2010
}

\section{Résumé}

Nous analysons l'impact de l'agriculture traditionnelle sur la diversité végétale dans une forêt semi-décidue afrotropicale non soumise à des perturbations naturelles majeures. Les assemblages d'espèces et les attributs structurels de la diversité sont décrits le long d'une chronoséquence sur 30 ans de champs abandonnés, comparativement aux communautés de forêts «primaires » et de forêts coupées sélectivement. Seulement 6,3\% des espèces inventoriées dans les forêts anciennes n'ont pas été observées dans les jachères, alors que 12,3\% des espèces sont propres aux jachères. Lorsque la forêt ancienne sur sol ferrallitique est prise comme référence, les analyses de régression linéaire montrent que cette forêt peut recouvrer la structure et la fonction au cours de la quatrième décennie de recolonisation, et la composition spécifique une décennie plus tard. L'invasion précoce des champs par Chromolaena odorata (L.) R. King \& H. Robinson. ne semble pas altérer durablement la succession secondaire. Les espèces s'assemblent en communautés végétales discrètes le long du gradient successionnel, en fonction de leurs exigences en lumière, suggérant une partition des niches écologiques.

Mots clés : Biodiversité végétale, successions secondaires, indices de diversité

\begin{abstract}
Plant biodiversity and dynamics of regeneration in a selective-logged forest (Sanaimbo, Ivory Coast)

This study aims at testing whether shifting cultivation contribute to plant diversity in an African semi-deciduous tropical forest lacking large-scale natural disturbance. For this purpose, I surveyed species assemblages and structural attributes of diversity along a 30 year-chronosequence of abandoned fields, comparatively to old-growth and selectively logged forest stands. Results show that only $6.3 \%$ of the species recorded in the old-growth forest are lacking in secondary stands, while $12.3 \%$ are restricted to fallows. When the old-growth forest on ferralitic soils is taken as the reference, regression analyses indicate that the old-growth forest may recover structure and function during the fourth decade of regrowth, and species composition only one decade later. Early colonization by the invasive Chromolaena odorata (L.) R. King \& H. Robinson does not appear to alter secondary succession. Species assembled themselves into discrete plant communities along the successional gradient with respect to their light requirements, suggesting niche partitioning.
\end{abstract}

Key-words: Plant biodiversity, secondary successions, indices of diversity 


\section{Introduction}

Les forêts tropicales humides de l'Afrique de l'Ouest constituent l'un des écosystèmes les plus diversifiés de la planète, avec un taux d'endémisme élevé (Myers et al., 2000). Elles font partie des forêts les plus menacées (Archard et al., 2002). Les forêts tropicales humides de l'Afrique de l'Ouest sont classées parmi les 25 " points chauds» de la biodiversité mondiale (c'est-à-dire, les écosystèmes les plus riches et les plus diversifiés de la planète) et constituent une priorité en matière de conservation (Myers et al., 2000 ; Puig, 2001). Les chablis et la théorie des perturbations intermédiaires permettent d'expliquer la haute biodiversité de ces forêts (Hubbell, 2001). Contrairement aux forêts indomalaises, les forêts africaines ne sont pas soumises à des perturbations à grande échelle (cyclones, grands incendies, éruptions volcaniques) (Jans et al., 1993). En Afrique tropicale, la croissance de la population et l'exploitation des terres agricoles qui en résulte est la principale cause de la destruction des forêts. Les forêts anciennes présentent une extraordinaire capacité de résilience puisqu'elles sont capables de retrouver une structure architecturale très proche de la forêt primaire quelques années seulement après l'abandon des terres agricoles, même si elle ne retrouve ni sa richesse spécifique ni sa composition floristique initiale (Chinea, 2002). L'impact des perturbations anthropiques dans les forêts tropicales a été mis en évidence récemment (Wright, 2005). Ces activités humaines ont des impacts locaux sur le milieu (tassement du sol, défrichements, faible enrichissement en matières organiques, ce qui modifie durablement la végétation en place). Des données récentes ont suggéré un rôle non négligeable des activités humaines du passé sur la biodiversité actuelle des forêts tropicales (van Gemerden, 2003). C'est le mythe de la forêt « vierge » qui s'émiette au fur et à mesure de l'accumulation des connaissances archéologiques dans les pays des régions tropicales (Willis et al., 2004). II est désormais admis que nombre d'entre elles ont été le siège d'une activité humaine relativement intense ne modifiant pas la structure ou la diversité spécifique, mais influençant la composition spécifique (Guariguata \& Ostertag, 2001). Les forêts primaires doivent être distinguées des forêts anciennes, qui sont de « vieilles » forêts secondaires (Denevan, 1992 ; Kassi \& Decocq, 2008) et serait, en fait, le produit de plusieurs siècles de succession secondaire suite à l'abandon des terres agricoles (Bush \& Colinvaux, 1994).

L'objectif de ce travail est de comprendre la distribution des espèces et leur assemblage en communautés végétales le long d'une chronoséquence de 30 ans, de jachères incluses dans une matrice de forêt semi-décidue. II s'agit également de vérifier que la forêt classée de Sanaimbo, considérée comme primaire, est capable de se régénérer après un épisode cultural. Plus spécifiquement, nous allons tenter de répondre à deux questions.

- Est-ce que les espèces s'assemblent en communautés pendant la succession secondaire?

- Comment l'organisation fonctionnelle des communautés évolue-t-elle le long de la chronoséquence?

\section{2. materiel et methode}

\subsection{Site d'étude}

L'étude s'est déroulée dans la forêt classée de Sanaimbo. Cette forêt, d'une superficie de 4322 ha, est située au Centre-Est de la Côte d'Ivoire $\left(6^{\circ} 20^{\prime}-6^{\circ} 26^{\prime} \mathrm{N}, 4^{\circ} 33^{\prime}-4^{\circ} 37\right.$ O; altitude moyenne : $120 \mathrm{~m})$. Il s'agit d'une forêt dense semi-décidue, appartenant au secteur mésophile (Guillaumet \& Adjanohoun, 1971). La végétation climacique est la forêt dense humide semi-décidue (White, 1986).

\subsection{Méthode}

Nous avons suivi la méthodologie phytosociologique sous son approche synusiale « intégrée » (Gillet et al., 1991). Un seul relevé a été effectué pour chaque jachère ou parcelle de forêt. A l'intérieur de chaque placette, nous avons réalisé un relevé phytosociologique sur une surface échantillon de $1500 \mathrm{~m}^{2}$. Une telle surface permet de prendre en compte les aires minimales des jachères de différents âges et les forêts (Senterre, 2005). 
Le plan d'échantillonnage a été conçu de manière à inclure des reliquats de forêt primaire non exploitée, des forêts villageoises uniquement exploitées pour leur bois d'œuvre et des jachères de différents âges, qui correspondent à des stades plus ou moins matures de la succession secondaire post-culturale ( forêt primaire non exploitée : n (nombre de relevé)=10 ; forêts villageoises uniquement exploitées pour leur bois d'œuvre : $n=6$; jachères de $3-4$ ans : $n=6$, jachères de 5 - 9 ans : $n=11$, jachères de 10-14 ans : $n=9$, jachères de 15- 19 ans : $n=15$, jachères de 20-24 ans : $n=14$, jachères de $25-30$ ans : $n=9$ ). La succession secondaire post-culturale a été étudiée le long d'une chronoséquence de 64 jachères et comparée à 16 relevés de forêts soit un total de 80 relevés. L'âge des jachères a été obtenu après enquête auprès des agriculteurs qui ont cultivé les champs concernés. La nomenclature des espèces suit Lebrun et Stork (1991-1997). La détermination des hauteurs des strates découle d'une analyse architecturale sur le terrain et des résultats d'auteurs précédents (Kassi \& Decocq, 2007) pour les strates arborescentes $\left(A_{1}\right.$ : arbres $>25 \mathrm{~m}$ et $\mathrm{A}_{2}$ : arbres de $15-25 \mathrm{~m}$ ); $\mathrm{S}_{1}$ : arbustes de 10 à $15 \mathrm{~m}_{\text {et }} \mathrm{S}_{2}$ : arbustes de 5 à $10 \mathrm{~m} ; \mathrm{S}_{3}$ : arbustes $<5 \mathrm{~m}$ et $\mathrm{H}$ : herbacées.

\subsubsection{Analyse des données}

\subsubsection{Assemblages d'espèces}

Nous avons construit une matrice floristique contenant les 80 relevés. Afin de rechercher si les espèces s'assemblaient en communautés discrètes le long de la chronoséquence, nous avons d'abord soumis cette matrice à une classification hiérarchique ascendante $(\mathrm{CHA})$ en utilisant la méthode de Ward et les distances euclidiennes relatives comme mesure de distance. Une analyse des espèces indicatrices (ISA ; Dufrêne \& Legendre, 1997) nous a permis de couper le dendrogramme et de choisir le nombre optimal de groupes. La significativité de chaque valeur indicatrice a été testée par l'approche de Monte Carlo (Dufour, 2006).

\subsubsection{Régressions linéaires}

Nous avons étudié l'évolution des attributs structurels de diversités ( $\mathrm{S}, \mathrm{J}^{\prime}, \mathrm{H}^{\prime}, \mathrm{D}, \mathrm{b}_{1}$ et $\mathrm{b}_{2}$ ) auxquels nous ajoutons la phytochorie et le type biologique. Ces différents paramètres ont été introduits dans des analyses de régression linéaire en fonction du temps, pour les 64 relevés de jachères : richesse spécifique $(S)$, indice de diversité de Simpson (D), indice de diversité de Shannon $\left(H^{\prime}\right)$, indice d'équitabilité de Pielou ou 'evenness'. La similarité intra-groupe désignée par $\beta\llcorner$ permet de mesurer la variabilité de la composition spécifique à chaque stade de la succession. Considérant la forêt sur sol ferrallitique comme état de référence (Kassi \& Decocq, 2007), nous avons calculé la similarité entre chaque groupe d'espèces de la chronoséquence et le groupe de forêts primaires $\left(\beta_{2}\right)$. Ces deux coefficients de similarité ont été calculés en utilisant l'indice de Sørensen. L'évaluation de ces différents paramètres permet de voir si la succession secondaire converge vers la forêt « primaire ».

\subsubsection{Organisation fonctionnelle}

Nous avons choisi un ensemble de traits de vie qui étaient déjà disponibles dans la littérature ou qui pouvaient être facilement mesurés sur le terrain (Tableau 1). Nous avons également inclus le type de phytochorie (sensu White, 1986) dans nos analyses, comme mesure de la spécialisation. Les proportions relatives de chaque trait de vie et de chaque phytochorie ont été calculées pour chaque groupe issu de la $\mathrm{CHA}$. Pour les 64 relevés de jachères, ces proportions ont été introduites dans des régressions linéaires contre le temps. Les proportions obtenues pour le groupe des jachères les plus âgées et pour les relevés de forêt primaire sur sol ferrallitique ont été comparées à l'aide du test non paramétrique U de Mann et Whitney (1947).

Toutes les analyses ont été effectuées à l'aide du logiciel PC-Ord $®$ v. 5 (McCune \& Mefford, 2001) pour les analyses multidimensionnelles (CHA et ISA) et du logiciel Statview ${ }^{\circledR}$ (Abacus, 1996) pour les tests statistiques univariés.

\section{Résultats}

\subsection{Assemblages d'espèces}

Les 80 relevés comptaient 365 plantes vasculaires parmi lesquelles 115 (31,5\%) étaient présentes dans moins de $10 \%$ des relevés. A ces espèces, on peut en ajouter 6 autres 
espèces cultivées mais souvent présentes dans les vieilles jachères (Theobroma cacao L., Coffea arabica L.) ou seulement dans les plus jeunes jachères (< 15 ans) : Musa sapientum L., Dioscorea alata L. et Manihot esculenta Crantz. Nous avons inventorié dans l'ensemble des jachères 340 espèces $(93,1 \%)$, parmi lesquelles $42(12,3 \%)$ n'ont pas été observées dans les relevés de forêts (exemples, Rauvolfia vomitaria Afzel. et Trema orientalis (L.) Blume). Dans les dix relevés de forêts anciennes, 287 espèces $(78,6 \%)$ ont été inventoriées, parmi lesquelles $18(6,3 \%)$ n'ont pas été observées dans les jachères (Didymosalpinx abbeokutae (Hiern) Keay et Amorphophallus johnsonii N. E. Br.).

La classification hiérarchique des 80 relevés, suivie de l'analyse des espèces indicatrices, ont permis de retenir 14 groupes en coupant le dendrogramme à une distance euclidienne relative de 12,0 (Fig. 1). Ces groupes coïncident parfaitement avec les 11 classes d'âges des jachères (I à XI) et les 3 types forestiers (XII : forêt villageoise exploitée; XIII : forêt ancienne sur sol ferrallitique et XIV : forêt ancienne sur sol hydromorphe). L'âge de la jachère est le principal déterminant des groupes $(H=78,1 ; p<0,0001)$, avec une séparation nette entre les jeunes jachères ( $<22$ ans) des autres jachères. Ces jeunes stades sont surtout des broussailles peu structurées, caractérisées par des espèces de la strate sousarbustive $\left(\mathrm{S}_{3}\right)$. Ils sont largement dominés par une espèce invasive, Chromolaena odorata (L.) R. King \& $\mathrm{H}$. Robinson, qui occupe à elle seule plus de $75 \%$ de la couverture végétale. Les quatre premiers groupes (I à IV) sont floristiquement semblables et riches en espèces héliophiles pionnières (exemples, Paullinia pinnata L. et Lantana camara L.). Les groupes $\mathrm{V}$ et $\mathrm{VI}$ sont caractérisés par l'apparition des espèces arbustives (exemples, Lannea welwitschii (Hiern) Engl. et Musanga cecropiodes $\mathrm{R}$. Br.), qui mettent en place la strate arbustive basse $\left(\mathrm{S}_{2}\right)$ et la strate arbustive haute $\left(S_{1}\right)$, entre la $10^{\text {ème }}$ et la $15^{\text {ème }}$ années après l'abandon des champs. De même, les groupes VII et VIII, qui englobent les jachères de 16-22 ans, sont caractérisées par de petits arbres héliophiles pionniers (Ficus exasperata Vahl et Trichilia monadelpha (Thonn.) De Wilde), des lianes (Salacia baumanii Loes.) et les premières espèces herbacées (Tragia vogelii Keay). La deuxième branche du cluster est surtout caractérisée par des espèces de la strate arborescente dominée $\left(A_{2}\right)$, exemples, Albizia adianthifolia (Schum.) W. F. Wright et $A$. zygia (DC.) J. F. Macbr. Les deux groupes de forêts secondaires ( $\mathrm{X}$ et $\mathrm{XI}, 23-30$ ans) sont clairement séparés des trois groupes de forêts anciennes (groupes XII, XIII et XIV). La composition floristique de ces vieilles jachères est caractérisée par des espèces cicatricielles longévives à croissance rapide (exemples, Albizia adianthifolia (Schum.) W. F. Wright et Ceiba pentandra (L.) Gaertn.) et de nombreuses lianes (Cissus aralioides (Welw. ex Bak.) Planch.). Les groupes de forêts anciennes possèdent le plus grand nombre d'espèces indicatrices. Elles sont caractérisées par les grands arbres $\left(A_{1}\right)$, exemples, Celtis zenkeri Engl. et Nesogordonia papaverifera (A. Chev.) Cap. de grandes lianes, exemple, Calycobolus africanus (G. Don) Heine et par des espèces sciaphiles de la strate sous-arbustive $\left(\mathrm{S}_{3}\right)$, exemples, Turraea heterophylla J. Sm. et Diospyros monbuttensis Gürke. 


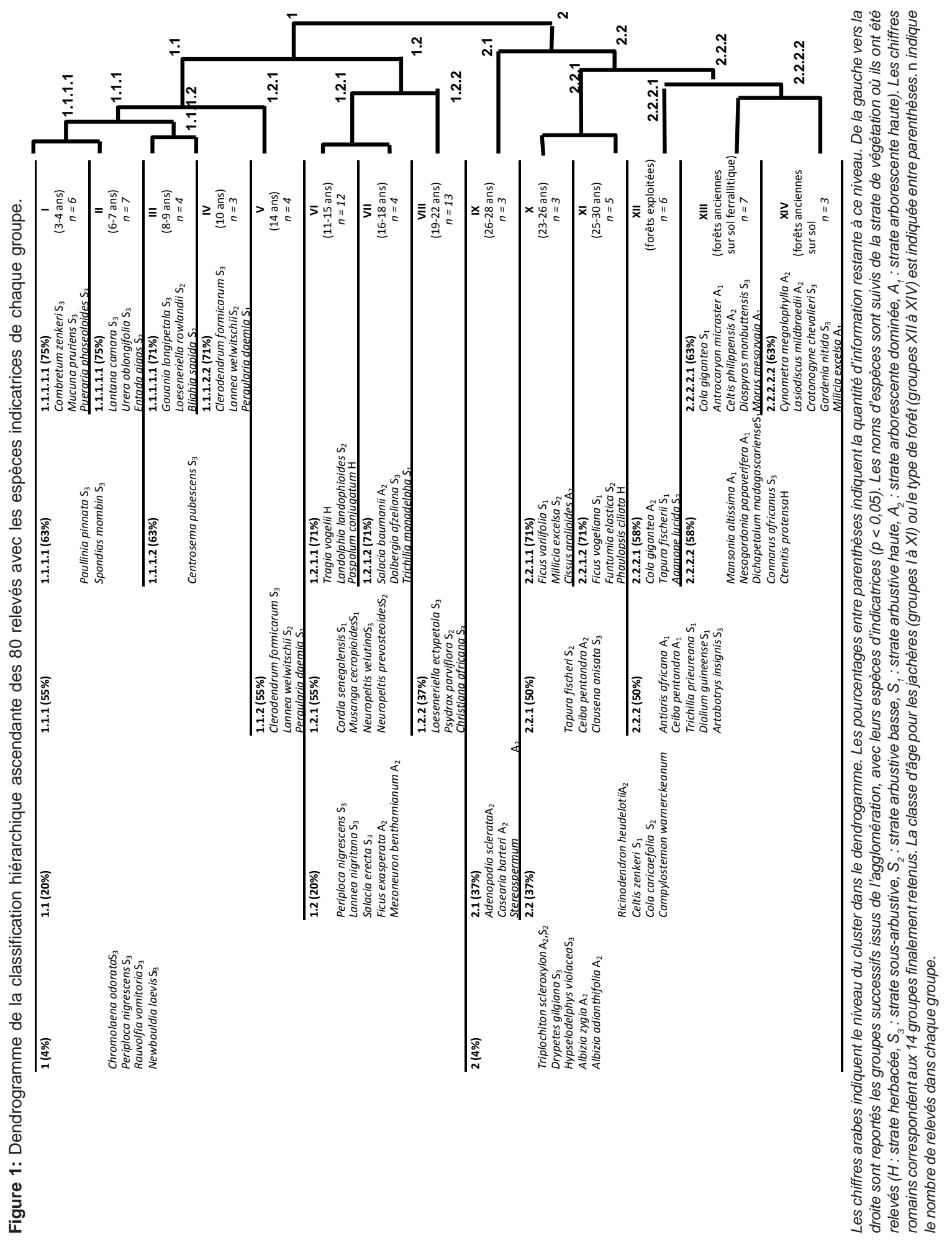




\subsection{Attributs structurels de la diversité}

Tous les paramètres de diversité sont fortement corrélés avec l'âge de la jachère (Fig. 2). La richesse spécifique augmente régulièrement avec l'âge le long de la chronoséquence, atteignant 141 espèces dans les jachères de 30 ans. Mais cette valeur

reste inférieure à celle observée en forêt ancienne (Tableau 1). D'après l'équation de la droite de régression linéaire de la richesse spécifique en fonction du temps, les jachères atteindraient la richesse spécifique des forêts anciennes 32 ans seulement après l'abandon des cultures. Les indices de Simpson (D) et de Shannon ( $\left.\mathrm{H}^{\prime}\right)$ augmentent régulièrement avec l'âge des jachères, puis atteignent une asymptote respectivement autour de 0,99 et de 4,7 vers l'âge de 15 ans (Fig. 3). Néanmoins, ils restent toujours inférieurs aux valeurs recensées dans les relevés de forêts anciennes non perturbées (Tableau 1).

L'indice d'équitabilité de Piélou augmente régulièrement jusqu'à l'âge de 20-25 ans, où il atteint un pic autour de 0,98 avant de diminuer légèrement à 0,96 . Cette dernière valeur est proche de celle observée en forêt ancienne.
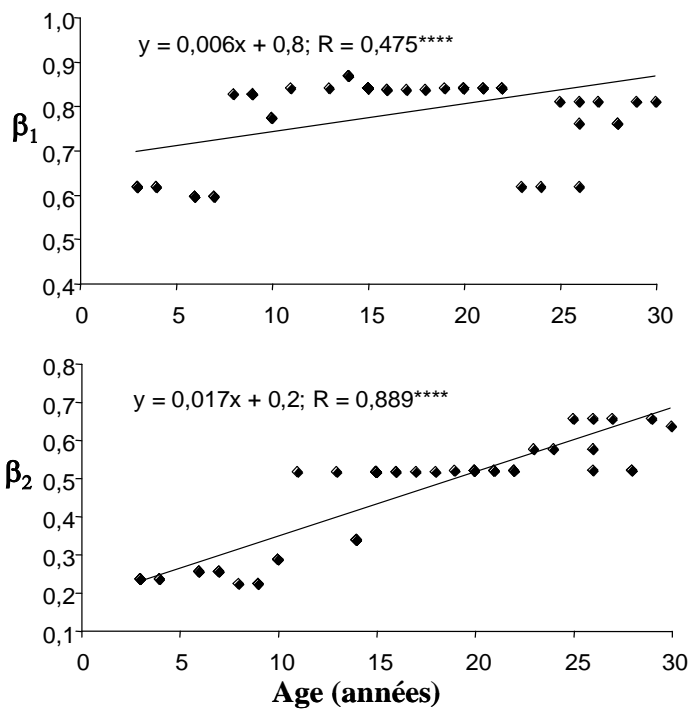

Figure 2 : Diagramme de régression linéaire des similarités floristiques intra-relevé $\left(\beta_{1}\right)$ et inter-relevés $\left(\beta_{2}\right)$ en fonction de l'âge des jachères $(n=64)$.
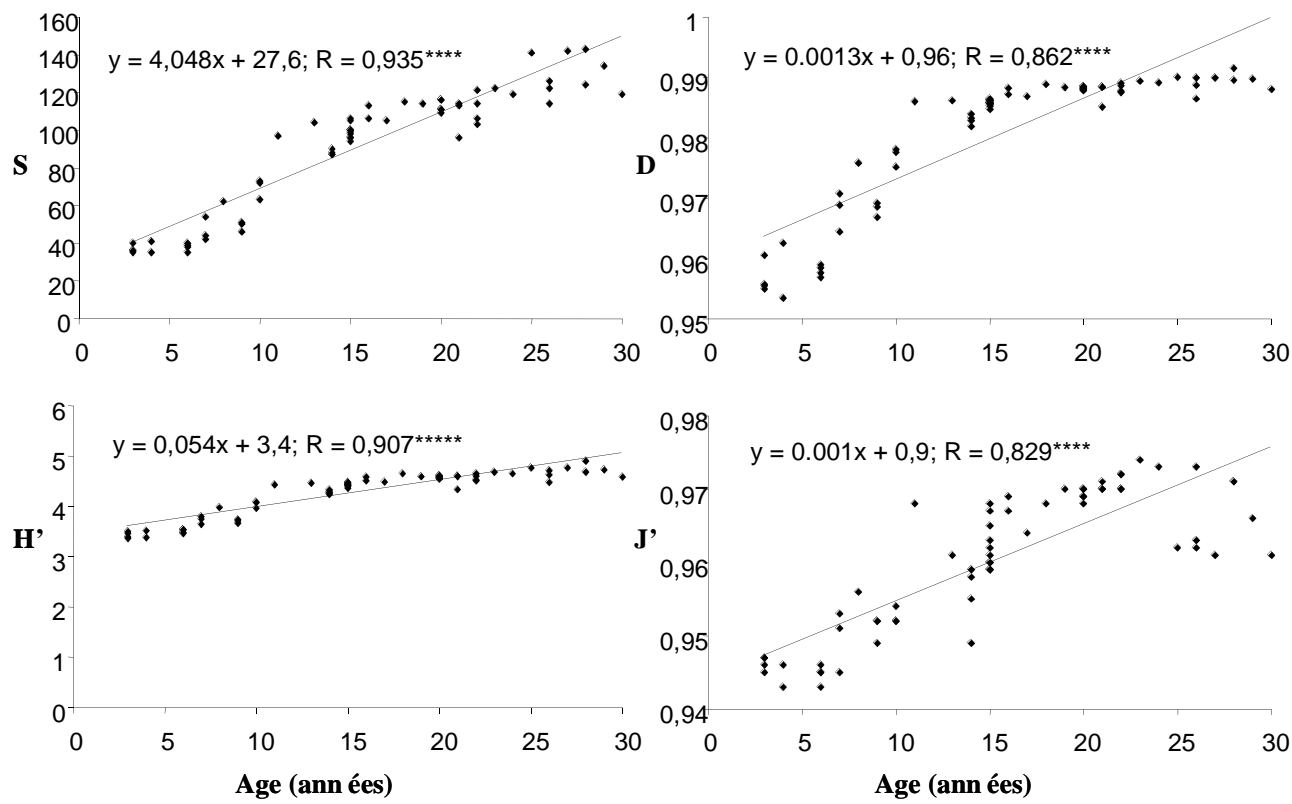

Figure 3 : Diagrammes des régressions linéaires des attributs structurels en fonction de l'âge des jachères $(n=$ 64). S : richesse spécifique, $\mathrm{D}$ : indice de diversité de Simpson, $\mathrm{H}^{\prime}$ : indice de diversité de Shannon, J' : indice d'équitabilité de Piélou. 


\subsection{Phytochories et type biologique}

On remarque qu'il y a une nette tendance à la spécialisation de la flore le long de la chonoséquence (Fig. 4). En début de succession, on note une proportion élevée des espèces à larges distributions (exemples, PA, Pan, Aam), qui diminue ensuite avec l'âge. II n'y a pas de différence significative entre le spectre des phytochories des jachères matures et celui des forêts anciennes (Tableau 1). Les endémiques guinéo-congolaises (exemples, GC, GCW) s'accumulent rapidement le long du gradient successionnel, à mesure que la richesse spécifique augmente, passant de moins de $40 \%$ dans les jeunes jachères à $60 \%$ dans les vieilles jachères. Les proportions des autres phytochories varient peu (AM, Pal, GC-S) ou pas (AT) le long de ce même gradient. La régression du type biologique en fonction de l'âge des jachères met en évidence qu'il n'y a pas de changement majeur du spectre des types biologiques le long de la chronoséquence, toutes les communautés végétales étant quantita- tivement dominées par les micro-phanérophytes, même si leur proportion diminue au cours du temps (Fig. 5). Les proportions de chaméphytes, de nano-, méso- et mégaphanérophytes varient peu le long de la chronoséquence. Les géophytes, les hémicryptophytes et les épiphytes, qui restent des types marginaux, n'apparaissent seulement qu'après 10,10 et 23 ans respectivement. Les thérophytes ne sont principalement présentes que dans les très jeunes jachères. Le spectre biologique des vieilles jachères est semblable à celui des forêts anciennes, avec toutefois une plus faible proportion de microphanérophytes et d'hémicryptophytes et une plus forte proportion de thérophytes, de chaméphytes et de mésophanérophytes (Tableau 1). Dans les jeunes jachères, jusqu'à $50 \%$ des espèces sont des lianes. Ce pourcentage a tendance à s'infléchir au cours du temps pour approcher celui des forêts anciennes (ca. 30\%). La proportion des espèces épineuses ne varie pas le long de la chronoséquence, environ $10 \%$ (Fig. 5), mais reste plus élevée que celle observée en forêt ancienne ( $7,5 \%$ en moyenne).

Tableau 1: Comparaison des attributs entre vieilles jachères $(n=5)$ et forêts anciennes sur sol ferrallitique $(n=7)$

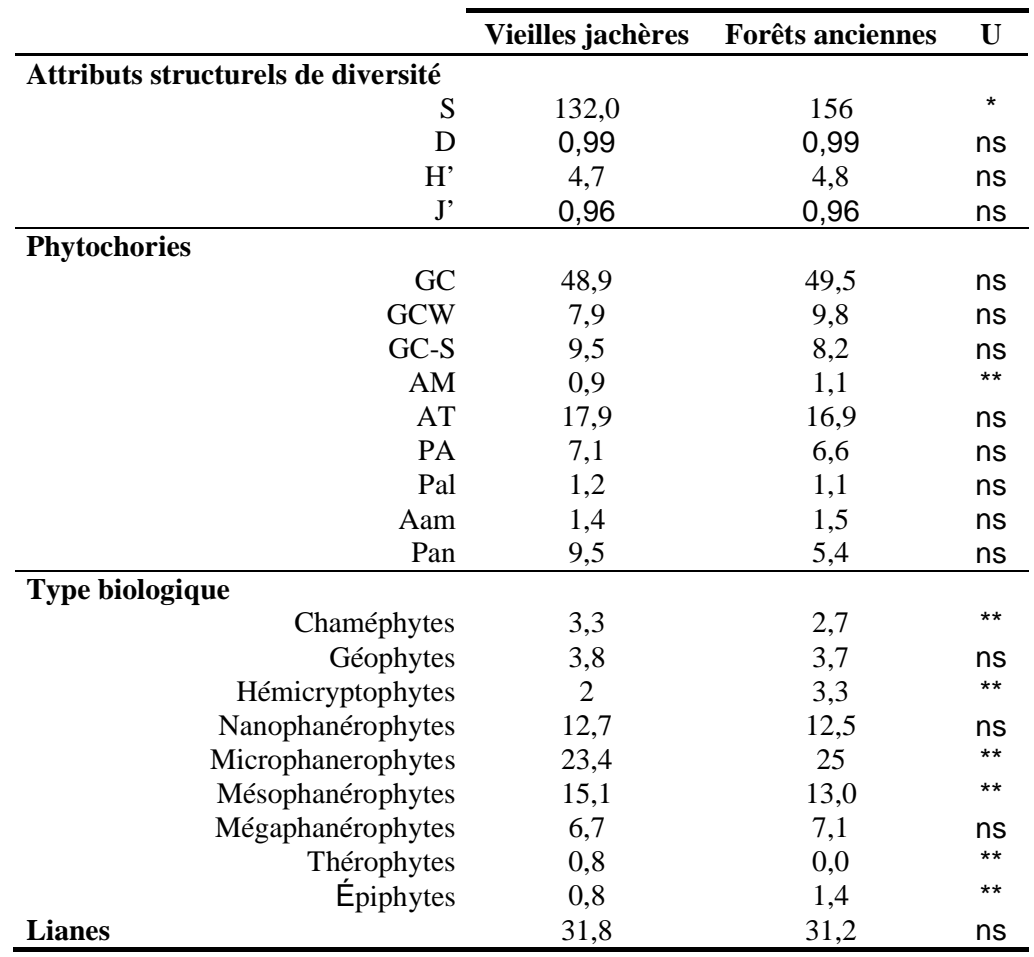

Les valeurs moyennes ainsi que les résultats du test de Mann \& Whitney (U) sont indiqués (ns: $p$ e» 0,05, *: $p<0,05, * *: p<0,01$ ). $S$ : richesse spécifique, $D$ : indice de Simpson, $H^{\prime}$ : indice de diversité de Shannon, J' : indice d'équitabilité de Pielou, GC: Guinéo-congolaises, GCW : Guinéo-Congolaises endémiques ouest-africaines, GC-S : zone de transition entre les secteurs Guinéo-congolais et Soudaniennes, AM : Afro-Malgaches, AT : Afrotropicales, PA : Pluri-régionales africaines, Pal : Paléotropicales, Aam : Afro-américaines, Pan : Pantropicales. 

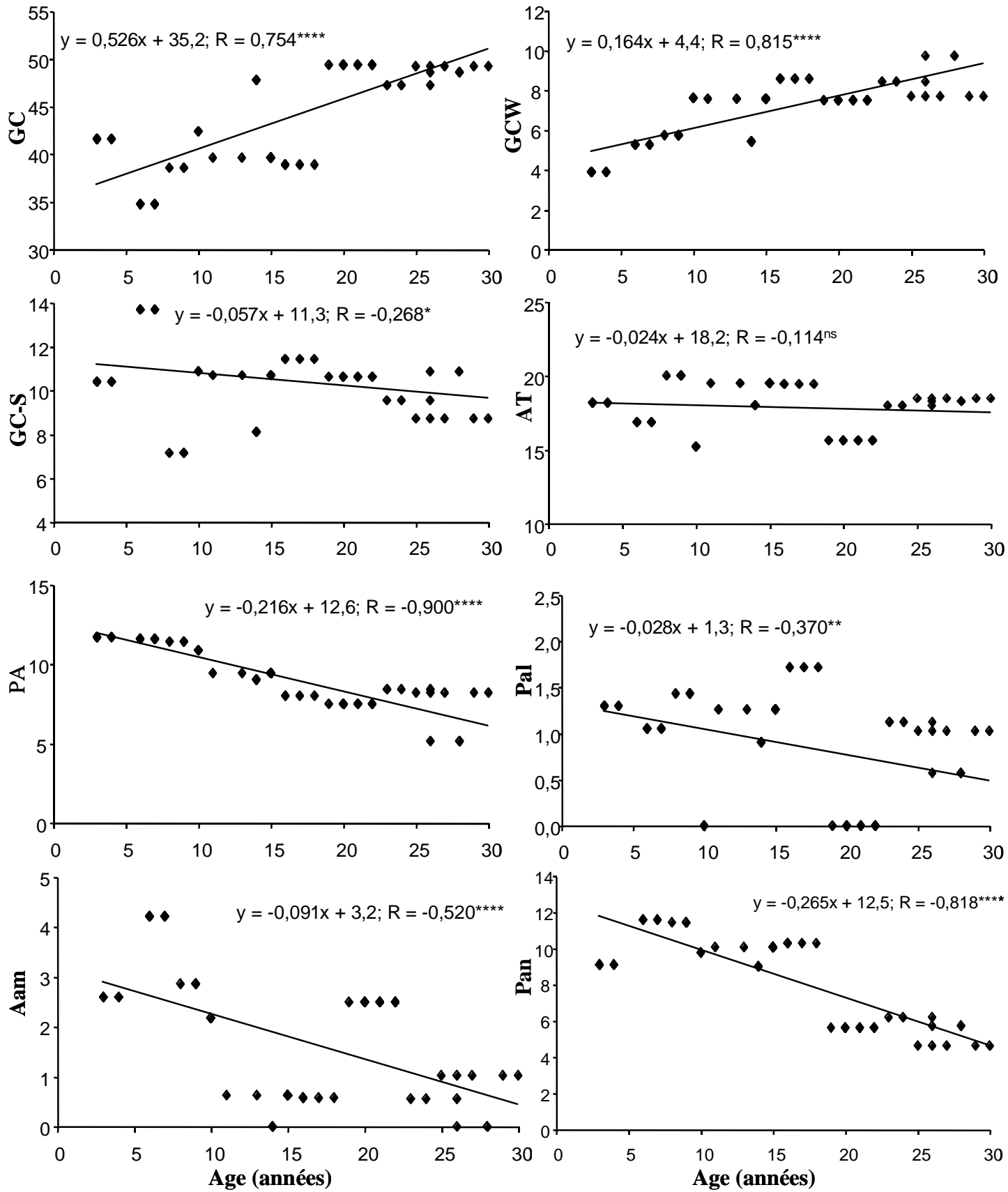

Figure 4: Diagrammes des régressions linéaires de la proportion des différentes phytochories en fonction de l'âge des jachères $(n=64)$. GC: Guinéo-congolaises, GCW : Guinéo-congolaises endémiques ouest-africaines, GC-S : zone de transition entre les régions Guinéo-Congolaises et Soudaniennes, AT : afrotropicales, PA : plurirégionales africaines, Pal : Paléotropicales, Aam: Afro-américaines, Pan : Pantropicales. 

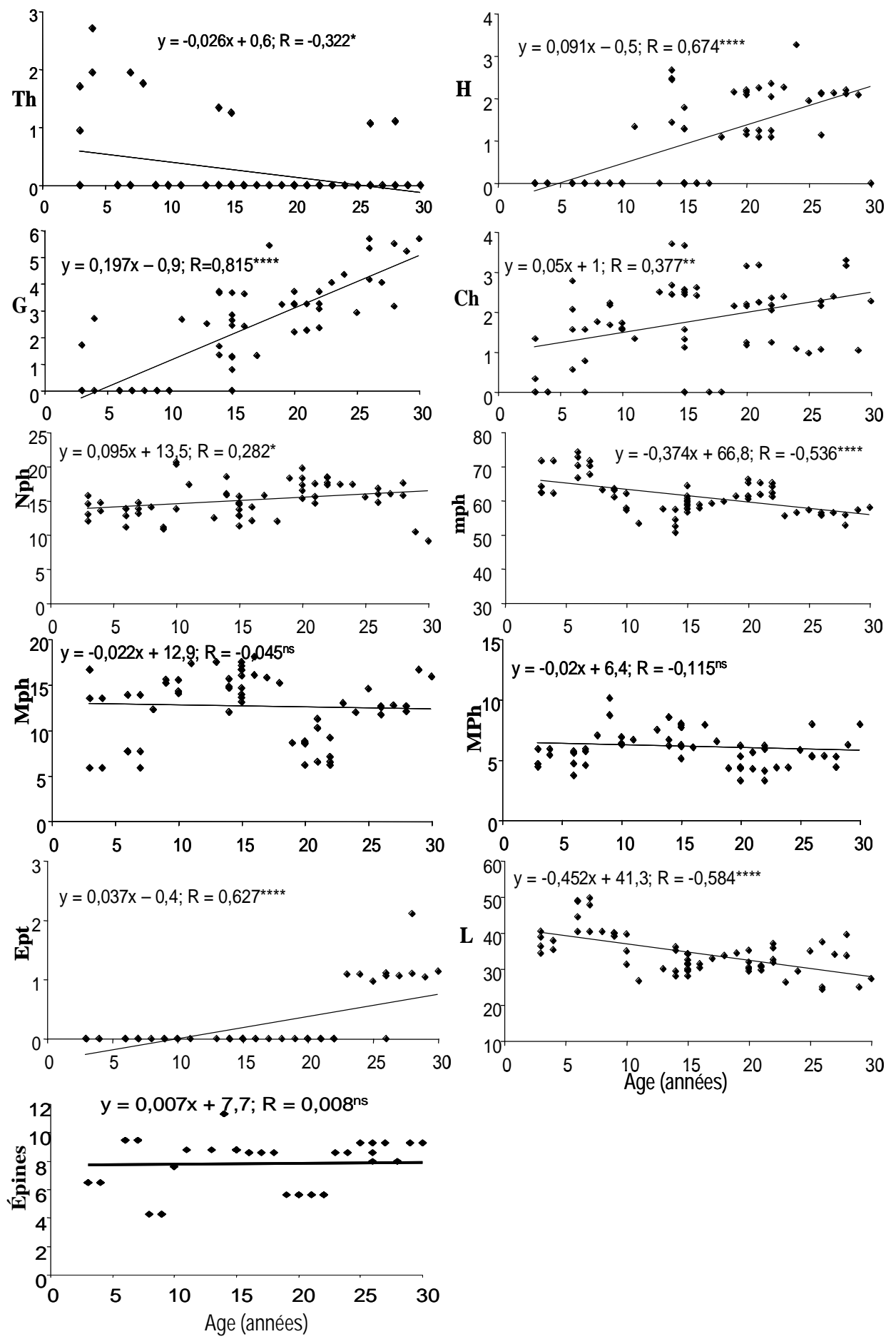

Figure 5 : Diagrammes des régressions linéaires des types biologiques en enfonction de l'âge des jachères $(n=64)$. Th : thérophytes, H : Hémicryptophytes, G : géophytes, Ch: Chaméphytes, Nph : nanophanérophytes, mph : microphanérophytes, Mph : mésophanérophytes, MPh : mégaphanérophytes, Eph : épiphytes, L: Lianes. 


\section{Discussion}

La forêt classée de Sanaimbo consiste en une mosaïque de forêts secondaires d'âge varié incluses dans une matrice de forêt ancienne. Nos résultats montrent clairement que l'âge de la jachère est le principal facteur expliquant la structuration des espèces en communautés végétales. II en explique également les organisations structurelle et fonctionnelle. Cela suggère que le processus d'assemblage des espèces est déterministe (Hubbell, 2001). Par conséquent, la composition floristique des communautés végétales post-culturales serait prédictible une fois le réservoir local d'espèces et l'âge des jachères connus, conformément aux règles de coexistence des espèces de la théorie des niches (Chinea, 2002 ; Guariguata \& Ostertag, 2001). Les végétations secondaires différant par leur âge offrent des conditions d'habitat et de ressources variées, en particulier en ce qui concerne la disponibilité en lumière dans le sousbois, de telle sorte que les niches écologiques et, donc, les assemblages d'espèces se différencient le long de la chronoséquence, permettant la coexistence de nombreuses espèces à l'échelle de cette dernière dans son ensemble (Kassi \& Decocq, 2008). Nous notons comme Rivera et al. (2000) une grande variabilité dans la composition floristique des jachères les plus jeunes par rapport aux jachères plus âgées, comme l'indiquent d'ailleurs les résultats de la $\mathrm{CHA}$ et les valeurs élevées de $b_{1}$.

En accord avec les données qui s'accumulent dans la littérature traitant des successions secondaires en région tropicale et les généralisations de plus en plus robustes qui en découlent (Finegan, 1996 ; Rivera et al., 2000 ; Guarigata \& Ostertag, 2001), nos résultats montrent bien qu'une fois qu'une clairière culturale est abandonnée, le développement d'une forêt secondaire est très rapide (environ 30 ans). Les équations des droites de régression obtenues suggèrent que la richesse spécifique et les attributs structurels de la diversité devraient égaler ceux des forêts anciennes dès la quatrième décennie de recolonisation. De même, les différentes proportions des types biologiques et de phytochorie approchent celles observées en forêt ancienne. L'ensemble de ces résultats suggère que l'organisation fonctionnelle et la structure de la forêt tropicale semi-décidue se rétablissent dans un délai de 30 à 40 ans après un épisode cultural (Kassi \& Decocq, 2008). La capacité de régénération de la forêt tropicale dense semi-décidue en Afrique occidentale est importante si les sources de propagules sont nombreuses et proches, et que les sites n'ont subi qu'une courte période de mise en culture. Dans ces conditions, la forêt ne mettrait seulement que quelques décennies pour se régénérer. Le système agricole traditionnel de cultures itinérantes pourrait apparaître comme un mode d'exploitation durable des terres si les champs sont de petites tailles, inclus dans une matrice forestière, que l'agriculture n'a duré que quelques années et qu'une mise au repos de plusieurs années sépare deux épisodes de mise en culture.

D'une façon générale, ces résultats suggèrent une très bonne résilience de la forêt dense semidécidue après un épisode cultural. Ils viennent donc nuancer des études antérieures (Turner et al., 997) qui suggéraient que la reconstitution postculturale des forêts tropicales n'était pas constante. Elle dépend, d'une part, de la nature et de l'intensité des pratiques agricoles antérieures, et, d'autre part, de l'environnement immédiat dans lequel se produit la succession (Chinea, 2002 ; Kassi \& Decocq, 2007). Les attributs structurels, comme la richesse spécifique, mettent jusqu'à 80 années après l'abandon des cultures pour retrouver leur valeur initiale (Guarigata \& Ostertag, 2001), mais la plupart des études n'ont pas permis d'objectiver une restauration des communautés végétales de la forêt « primaire » (Aubréville, 1947 ; Namur \& Guillaumet, 1978 ; Moutsamboté, 1985 ; Finegan, 1996)

\section{Conclusion}

Nous concluons que l'assemblage des espèces dans les jachères est conforme aux règles de la théorie des niches, qui prédisent un assemblage déterministe des espèces en communautés végétales le long du gradient successionnel, en particulier en fonction de leur exigence en lumière. Toutefois, le hasard peut jouer un rôle non négligeable dans les jeunes stades en raison des différences dans les capacités individuelles des espèces pionnières à se disperser et à persister. Ceci contribue à la biodiversité élevée de la forêt classée de Sanaimbo dans son ensemble, puisque 93,1\% des espèces du réservoir local ont été retrouvées 
dans l'ensemble des jachères et $12,3 \%$ des espèces leur étaient propres parce que non observées dans les relevés de forêt ancienne considérée comme « primaire ».

\section{Remerciements}

Nous remercions le Prof. Laurent AKE ASSI pour son aide à l'identification des échantillons d'herbier et à la vérification de toutes nos déterminations ainsi que Feu le Dr. Georges AMAN KADIO qui nous a aidé à mener à bien les travaux de terrain.

\section{Références citées}

Abacus C., 1989. Statview 1.1 In Berkeley C. \& Anderson A.B., 1978. The names and uses of palms among a tribe of Yanomama Indians. Principes 22: 30-41.

Aubréville A., 1947. Les brousses secondaires en Afrique Equatoriale (Côte d'Ivoire, AEFCameroun). Bois Forêt Trop. 2: 24-49.

Archard F., Eva H.D., Stibig H.J., Mayaux P., Gallego J., Richards T. \& Mallingreau J.P., 2002. Determination of deforestation rates of the World's humid tropical forests. Science 297: 999-1002.

Bush M.B. \& Colinvaux P.A., 1994. Tropical forest disturbance: paleoecological records from Darién, Panamá. Ecology 75: 1761-1768.

Chinea J.D., 2002. Tropical forest succession on abandoned farms in the Humacao Municipality of eastern Puerto Rico. Forest Ecol. Manage. 167: 195-207.

Denevan W.M., 1992. The pristine myth: the landscape of the Americas in 1942. An. Ass. Am. Geogr. 82: 369-385.

Dufrêne M. \& Legendre P., 1997. Species assemblages and indicator species: the need for a flexible asymmetrical approach. Ecol. Monogr. 67: 345-366.

Dufour J.-M., 2006. Monte Carlo test with nuisance parameters: Ageneral approach to finite-sample inference and non-standard asymptotics in econometrics. J. Econ. 133: $443-477$
Finegan B., 1996. Pattern and process in neotropical secondary rain forest: the first 100 years of succession. Trends. Ecol. Evol. 11: 119-124.

Gillet, F., de Foucault, B. \& Julve, P. (1991). La phytosociologie synusiale intégrée : objet et concepts. Candollea 46: 315-340.

Guariguata M.R. \& Ostertag R., 2001. Neotropical secondary forest succession: changes in structural and functional characteristics. Forest Ecol. Manage. 148: 185-206.

Guillaumet J.-L. \& Adjanohoun E., 1971. La végétation de la Côte d'Ivoire. In : Le milieu naturel de la Côte d'Ivoire. Mémoires de l'ORSTOM 50: 157-263.

Hubbell S.P., 2001. The unified neutral theory of biodiversity and biogeography. Princeton University Press, Princeton, 375 pp.

Jans L., Poorter L., Van Rompaey R.S.A.R. \& Bongers F., 1993. Gaps and forest zones in tropical moist forest in Ivory Coast. Biotropica 25: $258-269$

Kassi N'.J. \& Decocq G., 2008. Spatio-temporal patterns of plant species and community diversity in a semi-deciduous tropical forest under shifting cultivation. J. Veg. Sci. 9 (6): 809-820.

Kassi N'.J. \& Decocq G., 2007. Succession secondaire post-culturale en système forestier tropical semi-décidu de Côte d'Ivoire: approche phytosociologique intégrée et systémique. Phytocoenologia 37 (2): $175-219$

Lebrun J.P. \& Stork A.L., 1991-1997. Enumération des plantes à Fleurs d'Afrique Tropicale. Conservatoire et Jardin Botaniques de la Ville de Genève, Genève. Vol. 1 (249 pp.), vol. 2 (257 pp.), vol. 3 (341 pp.), vol. 4 (711 pp.).

Mann H.B. \& Whitney D.R., 1947. On a test of whether one of two random variables is stochastically larger than other. An. Math. Stat. 18: 50-60

McCune B. \& Mefford M.J., 2001. PC-Ord. Multivariate analysis of ecological data, version 4. MjM Software Design, Gleneden Beach, $238 \mathrm{pp}$ 
Moutsamboté J.-M., 1985. Dynamique de la reconstitution de la forêt Yombe (Domonika, $R$. P. Congo). Thèse de Doctorat, Université Bordeaux III, 299 pp.

Myers N., Mittermeier R.A., Mittermeier C.G., da Fonseca G.A.B. \& Kent J., 2000. Biodiversity hotsposts for conservation priorities. Nature 403: 853-858.

Namur C. (De) \& Guillaumet J.L. 1978. Grands traits de la reconstitution dans le Sud-Ouest ivoirien. Cahiers de l'ORSTOM, série Biologie XIII, n³: 197-201.

Puig H., 2001. La forêt tropicale humide. Belin, Paris, 448 pp.

Rivera L.W., Zimmerman, J.K. \& Aide, T.M., 2000. Forest recovery in abandoned agricultural lands in a karst region of the Dominican Republic. Plant. Ecol. 148: 115-125.

Senterre B., 2005. Recherches méthodologiques pour la typologie de la végétation et la phytogéographie des forêts denses d'Afrique tropicale. Résumé de Thèse, Acta. Bot. Gall. 152: $409-419$.

Turner I.M., Wong Y.K., Chew P.T. \& bin Ibrahim A., 1997. Tree species richness in primary and old secondary tropical forest in Singapore. Biodiv. Conserv. 6: 537-543.

Van Germerden B., Olff H., Parren M.P.E. \& Bongers F., 2003. The pristine rain forest? Remnants of historical human impacts on current tree species composition and diversity. J. Biogeogr. 30: 1331-1390.

White F., 1986. La végétation de l'Afrique. Recherche sur les ressources naturelles. ORSTOM-UNESCO, Paris, XX, 384.

Wright S.J. 2005. Tropical forests in a changing environment. Trends. Ecol. Evol. Vol. 20: $553-560$.

Willis, K.J., Gillson, L. \& Brncic, T.M., 2004. How «Virgin» is Virgin rainforest? Science 304: 402-403. 